TITLE: Phase Stability and Elasticity of C15 Transition-Metal Intermeta11ic Compounds

AUthoR(S): F. Chu, T.E. Mitchell, S.P. Chen, M. Sob, R. Siegl, and D.P. Pope

SUBMITTED to: 1995 TMS ANNUAL MEETING

LAS VEGAS, NV U.S.A.

FEBRUARY 12-16, 1995 


\title{
Phase Stability and Elasticity of C15 Transition-Metal Intermetallic Compounds
}

\author{
F. Chu, T. E. Mitchell, and S. P. Chen \\ Los Alamos National Laboratory, Los Alamos, NM 87545, U. S. A. \\ M. Sob*, R. Siegl, and D. P. Pope \\ Department of Materials Science and Engineering, University of Pennsylvania, Philadelphia, \\ PA 19014, U. S. A.
}

\begin{abstract}
$\underline{\text { Abstract }}$
First-principle quantum mechanical calculations based on the local-density-functional theory have been performed to study the electronic, physical and metallurgical properties of C15 intermetallics $\mathrm{MV}_{2}\left(\mathrm{M}=\mathrm{Zr}\right.$, Hf, or Ta). The elastic constants of $\mathrm{C} 15 \mathrm{HfV}_{2}+\mathrm{Nb}$ were measured by the resonant ultrasound spectroscopy technique. The phase stability of $\mathrm{C} 15 \mathrm{HfV}_{2}+\mathrm{Nb}$ was studied by specific heat measurements and by transmission electron microscopy in a low temperature specimen holder. The total energies and their lattice volume dependence were used to obtain the equilibrium lattice constants and bulk modulus. The band structures at the X-point near the Fermi level were employed to understand the anomalous temperature dependence of shear modulus of the $\mathrm{C} 15$ intermetallics. It was found that the double degeneracy with a linear dispersion relation of electronic levels at the $\mathrm{X}$-point near the Fermi surface is mainly responsible for the $\mathrm{C} 15$ anomalous elasticity. The density of states at the Fermi level, $N\left(E_{F}\right)$, and the Fermi surface geometry were obtained to understand the low temperature phase instability of $\mathrm{C} 15 \mathrm{HfV}_{2}$ and $\mathrm{ZrV}_{2}$ and the stability of $\mathrm{C} 15 \mathrm{TaV}_{2}$. It was proposed that the large $N\left(E_{F}\right)$ and Fermi surface nesting are the physical reasons for the structural instability of the C15 $\mathrm{HfV}_{2}$ and $\mathrm{ZrV}_{2}$ at low temperatures. The relationship between the anomalous elasticity and structural instability of $\mathrm{C} 15 \mathrm{HfV}_{2}$ and $\mathrm{ZrV}_{2}$ were also discussed.
\end{abstract}

\section{DISCLAIMER}

\begin{abstract}
This report was prepared as an account of work sponsored by an agency of the United States Government. Neither the United States Government nor any agency thereof, nor any of their employees, makes any warranty, express or implied, or assumes any legal liability or responsibility for the accuracy, completeness, or usefulness of any information, apparatus, product, or process disclosed, or represents that its use would not infringe privately owned rights. Reference herein to any specific commercial product, process, or service by trade name, trademark, manufacturer, or otherwise does not necessarily constitute or imply its endorsement, recommendation, or favoring by the United States Government or any agency thereof. The views and opinions of authors expressed herein do not necessarily state or reflect those of the United States Government or any agency thereof.
\end{abstract}

* Permanent address: Institute of Physics of Materials, Academy of Sciences of the Czech Republic, Brno, Czech Republic. 


\section{DISCLAIMER}

Portions of this document may be illegible in electronic image products. Images are produced from the best available original document. 
Intermetallic compounds have been attractive candidates for high temperature structural applications because of their desirable intrinsic properties [1]. There have been many investigations carried out on various compounds, but mostly on structures that are ordered forms of fcc, bcc and hep metals. If new intermetallics are to be selected on the basis of low density and high melting temperature, then the attraction of two groups of materials becomes apparent: topologically close-packed (TCP) compounds [2] and silicide-based compounds [3].

In the group of TCP intermetallics, the Laves phases constitute the single largest group. Laves phases have either the cubic $\mathrm{C} 15\left(\mathrm{MgCu}_{2}\right)$, hexagonal $\mathrm{C} 14\left(\mathrm{MgZn}_{2}\right)$ or mixed C36(MgNi2) structure. Generally speaking, they have high melting temperatures and fairly low densities, and therefore could be attractive candidates for high temperature structural materials if their toughness could be increased at low temperatures. Among three Laves phases, C15 Laves phases are expected to show better deformability than the other two Laves phases because of their fcc based structure. For C15 Laves phases, early transition metal compounds, e.g., $\mathrm{HfV}_{2}+\mathrm{Nb}$, have been very interesting and subjected to comprehensive experimental studies [45], because they show high melting temperature, high strength and low temperature deformability. Other C15 Laves phase alloys have also been the subject of recent experimental studies, for example, $\mathrm{NbCr}_{2}$ [6-7], $\mathrm{HfCr}_{2}$ [8-9], $\mathrm{ZrFe}_{2}$ [10] and $\mathrm{TiCr}_{2}$ [11].

In order to improve the mechanical properties of this C15 alloy, it is necessary to understand its fundamental physical properties, e.g., phase equilibria, phases stability, elastic properties, and thermal properties.

Stability of transition metal Laves phases has been subjected to both theoretical and experimental studies $[12,13,14]$. The different roles played by size and electronic factors in stabilizing the transition metal Laves phases and the martensitic transformation associated with the transition metal Laves phases have been investigated particularly. Elastic properties of Laves phases are important because they relate to various fundamental solid-state phenomena such as interatomic potentials, equations of state, and phonon spectra. Elastic properties are also linked thermodynamically with specific heat, thermal expansion, Debye temperature, and Gruneisen parameter. Most importantly, knowledge of elastic constants is essential for many practical applications related to the mechanical properties of Laves phases as well: loaddeflection, thermoelastic stress, internal strain (residual stress), sound velocities, dislocation core structure and fracture toughness. This paper summarizes our work on phase stability and elastic properties of $\mathrm{C} 15$ intermetallic compounds $\mathrm{HfV}_{2}+\mathrm{Nb}$, including some of our results which have been reported.

\section{$\underline{\text { Review of Previous Work }}$}

For the binary $\mathrm{C} 15$ Laves phase compound $\mathrm{HfV}_{2}$, the phase stability and elastic properties have been studied. In the investigation of phase stability, a low temperature phase transformation, occurring around $110 \mathrm{~K}$, in $\mathrm{HfV}_{2}$ has been reported by a variety of experiments, e.g., neutron diffraction [15], elastic constants measurement [16] and specific heat measurement [17]. For the examination of elastic properties, it has been found that $\mathrm{C} 15 \mathrm{HfV}_{2}$ shows anomalous elastic behavior at both low and high temperatures (even up to the melting temperature), i.e., the shear and Young's moduli of $\mathrm{C} 15 \mathrm{HfV}_{2}$ increase with increasing temperature [18]. Especially, near the phase transformation temperature, there is a V-shaped softening of the shear and Young's modulus.

To understand the mechanism(s) of the low temperature phase instability and anomalous elasticity and the relationship between the two phenomena for C15 $\mathrm{HfV}_{2}$, theoretical approach has been performed based on the electronic structure consideration. It has been proved that for any $\mathrm{C} 15$ compound there is a double degenerate electronic energy levels with a linear dispersion relation at the $\mathrm{X}$-point in the irreducible Brillouin zone (IBZ), because of the crystal symmetry of $\mathrm{C} 15(\mathrm{Fd} 3 \mathrm{~m})$ [19]. It was also hypothesized that for the $\mathrm{C} 15 \mathrm{HfV}_{2}$, the Fermi level is filled by electrons in such a way that it just passes through the double degenerate point [19]. If this is true, it can be proved that the negative electronic contribution from these two bands to the 
trigonal shear modulus, $c_{44}^{e}$, is anomalous in the entire temperature region. It was suggested that this is the physical mechanism of the observed anomalous elasticity for C15 HfV 2 . Based on this suggestion, the $\mathrm{HfV}_{2}$ phase instability mechanism was rationalized as the result of the total $c_{44}$ softening, resulting from the cancellation of the negative and anomalous electronic contribution $\left(c_{44}^{e}\right)$ to the positive and normal ionic contribution $\left(c_{44}^{i}\right)$ [19]. Therefore, the C15 structural instability is directly related to the $c_{44}$ anomaly induced by the electronis effect.

The questions remaining in these studies are (1) Is it necessarily true that the Fermi surface of C15 HfV 2 exactly passes through the double degenerate point ? (b) If this is not true, how to understand the anomalous elastic properties of $\mathrm{Cl} 5 \mathrm{HfV}_{2}$ ? and (c) If this is not true, what is the physical mechanism for the low temperature phase instability of $\mathrm{C} 15 \mathrm{HfV}_{2}$ ? Bearing these questions in mind, we studied the phase stability and elastic properties of transition metal C15 Laves phases by a combination of experimental and theoretical approaches, using the $\mathrm{C} 15 \mathrm{HfV}_{2}+\mathrm{Nb}$ as an example.

\section{Experimental Procedure and Calculation Method}

Arc-melted buttons were used in the studies of phase stability and elastic properties of $\mathrm{C} 15 \mathrm{HfV}_{2}+\mathrm{Nb}$. The arc-melted buttons were made using elemental $\mathrm{Hf}, \mathrm{V}$, and $\mathrm{Nb}$ with nominal purities $99.99 \%, 99.9 \%$ and $99.7 \%$, respectively. For examining the C15 phase stability, five single phase samples, with compositions of $\mathrm{V} / \mathrm{Hf}=2$ and $\mathrm{Nb}$ at. $\%=0.0,0.5,1.0$, 2.0 , and 5.0, respectively, were chosen in this study based on the Hf-V-Nb ternary phase diagram [20]. The samples cut from arc-melted buttons were homogenized at $1200^{\circ} \mathrm{C}$ for $120 \mathrm{~h}$ in an argon atmosphere in order to produce a high degree of atomic ordering. The specific heat, $\mathrm{C}_{\mathrm{p}}$, was measured from $15 \mathrm{~K}$ to $250 \mathrm{~K}$ to investigate any phase transformation, in steps of $0.3 \mathrm{~K}$, using the semi-adiabatic heat pulse method in zero field in a closed cycle refrigeration system. Further examination on the low temperature structural transformation was performed by transmission electron microscopy, using a CM-30 Philip and in a low temperature specimen holder. We also measured the elastic constants of the polycrystalline $\mathrm{C} 15 \mathrm{Hf}_{25} \mathrm{~V}_{60} \mathrm{Nb}_{15} \mathrm{using}$ the resonant ultrasound spectroscopy technique (RUS) [21]. The polycrystalline $\mathrm{Hf}_{25} \mathrm{~V}_{60} \mathrm{Nb}_{15}$ specimen was cut into a rectangular parallelepiped with dimensions $a=2.086 \mathrm{~mm}, b=1.886 \mathrm{~mm}$, and $c=1.449 \mathrm{~mm}$. The mass-density of the specimen was determined from its dimensions and mass. To study the temperature dependence of the elastic properties of the ternary C15 compound, the measurements were carried out from room temperature to $70 \mathrm{~K}$, using boil-off gas of a liquid helium storage dewar and a temperature controller [22].

In the first-principle calculations, the total energy and the electronic structure of the C15 phases $\mathrm{MV}_{2}(\mathrm{M}=\mathrm{Zr}$, Hf, or Ta) were determined self-consistently using the linear muffin-tin orbital (LMTO) method with the atomic sphere approximation (ASA). The C15 (AB $)_{2}$ structure, as a TCP structure with the space group $\mathrm{Fd} 3 \mathrm{~m}\left(O_{h}^{7}\right)$, has $71 \%$ of the volume filled by touching spheres and high site symmetry for the two constituent elements. These facts make the C15 phase suitable for studies with the LMTO method using the atomic sphere approximation (ASA). The basis set included $s, p$ and $d$ orbitals $\left(l_{\max }=2\right)$ for each site. The self-consistent iterations used 14 points in reciprocal $\mathbf{k}$ space along $\Gamma$-X direction in the irreducible Brillouin zone (IBZ). The total energy and the electronic band structure were determined at 372 independent $\mathbf{k}$ points within the IBZ. The overlapping Wigner-Seitz (WS) spheres used for these LMTO (ASA) calculations were chosen by scaling touching muffin-tin (MT) spheres so that the total volume of all spheres equals that of the unit cell and the ratio rule of the C15 Laves phase, $r_{A} / r_{B}=(3 / 2)^{1 / 2}$, is satisfied. Consequently, the WS spheres are $0.2426 a_{o}$ for $M(M=Z r$, $\mathrm{Hf}$ and $\mathrm{Ta}$ ) sites and $0.1981 \mathrm{a}_{0}$ for $\mathrm{V}$ sites, where $\mathrm{a}_{\mathrm{o}}$ is the experimental lattice parameter. The valence states were treated in a semi-relativistic scheme, and the spin-orbit coupling term was neglected for the early transition metal elements. The core states were treated fully relativistically. The exchange and correlation potential used in the calculations was the von Barth-Hedin local density formalism. The details of the calculation were presented in Ref. [23]. The electronic energy eigenvalues at $372 \mathrm{k}$ points of the IBZ and LMTO parameters obtained from the equilibrium configuration were employed to determine the final band structures, densities of states and the Fermi surfaces of the C15 phases. 


\section{C15 Phase Stability}

Using the phase diagram of the Hf-V-Nb system [20], the phase stability of the C15 intermetallic compound based on $\mathrm{HfV}_{2}$ was studied. Sensitive specific heat measurements from the five single phase samples, with the compositions of $\mathrm{V} / \mathrm{Hf}=2$ and $\mathrm{Nb}$ at. $\%=0.0,0.5,1.0$, 2.0, and 5.0, respectively, at low temperatures are shown in Fig. 1. Figure 1 reveals that the binary $\mathrm{C} 15$ Laves phase $\mathrm{HfV}_{2}$ is unstable at low temperatures. A structural phase transformation occurs at about $115 \mathrm{~K}$. This is basically consistent with previous work on $\mathrm{HfV}_{2}$, e.g., neutron diffraction [15], elastic constants measurement [16] and specific heat measurement [17]. Further transmission electron microscopy study using a liquid nitrogen stage unambiguously shows that this is indeed a structural transformation, because of the appearance of superlattice spots in diffraction patterns as shown in Fig. 2. A detailed analysis of the diffraction patterns of different zone axis is in progress, by which the crystal symmetry of the low temperature phase could be revealed. Most importantly, Fig. 1 reveals in the first time that $\mathrm{Nb}$ ternary alloying can reduce the transition temperature and heat absorption in the structural transformation of the C15 Laves phase $\mathrm{HfV}_{2}+\mathrm{Nb}$, and in fact small $\mathrm{Nb}$ additions of 2-3 at.\% eliminate this structural phase transformation entirely. This means that the role played by the $\mathrm{Nb}$ ternary alloying is to stabilize the structure of $\mathrm{C} 15$.



Figure 1. Heat capacity vs. temperature curves for five ternary C15 Laves compounds based on $\mathrm{HfV}_{2}$ in the vicinity of the lattice transformation temperature.

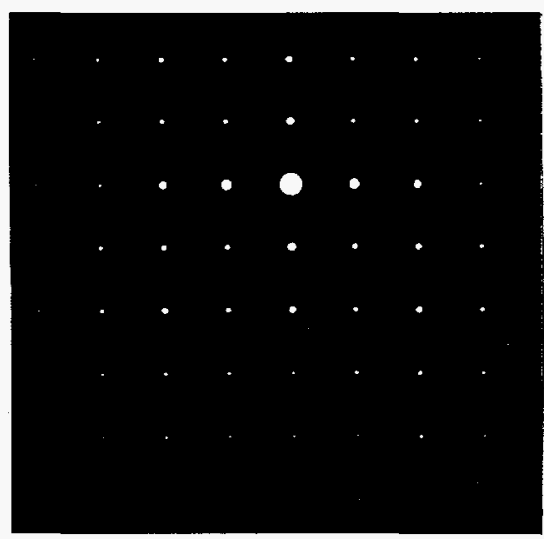

(a)

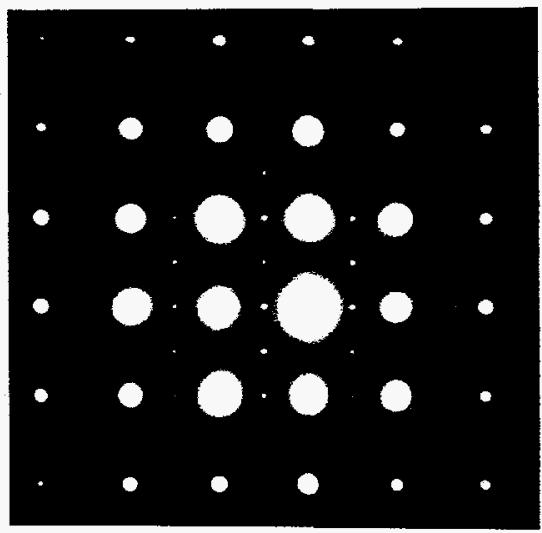

(b)

Figure 2. Diffraction patterns of $\mathrm{HfV}_{2}$ along $\{100\}$ zone axis: (a) at room temperature, and (b) at $83 \mathrm{~K}$, showing the superlattice spots after the structural transformation in $\mathrm{HfV}_{2}$. 


\section{Elastic Properties}

The shear modulus, Young's modulus, Bulk modulus and Poisson's ratio of the ternary $\mathrm{C} 15 \mathrm{Hf}_{25} \mathrm{~V}_{60} \mathrm{Nb}_{15}$ are shown as a function of temperature in Fig. 3. It can be seen from Fig. 3 that the shear and Young's moduli of $\mathrm{C} 15 \mathrm{Hf}_{25} \mathrm{~V}_{60} \mathrm{Nb}_{15}$ are anomalous, i.e., they increase with increasing temperature. The bulk modulus is virtually constant, and the Poisson's ratio (above 0.4 ) is higher than observed for most materials and decreases with increasing temperature. These results indicate that an anomaly in elastic properties still exists in ternary C15 compounds, although there is no low temperature structural instability in the ternary compounds. This suggests that the shear modulus anomaly is not directly related to such a phase transformation in $\mathrm{C} 15$ transition metal Laves phases.


Figure 3. Temperature dependence of elastic constants of $\mathrm{C} 15 \mathrm{Hf}_{25} \mathrm{~V}_{60} \mathrm{Nb}_{15}$ : (a) shear modulus $\mathrm{G}$ vs. $\mathrm{T}$, the error is $\pm 0.02 \mathrm{GPa}$, (b) Young's modulus $\mathrm{E}$ vs. $\mathrm{T}$, the error is $\pm 0.13 \mathrm{GPa}$, (c) bulk modulus $\mathrm{B}$ vs. $\mathrm{T}$, the error is $\pm 0.87 \mathrm{GPa}$, and (d) Poisson's ratio v vs. T, the error is \pm 0.001 (from Ref. [22]).

\section{Total Energy and Electronic Structure of $\mathrm{C} 15 \mathrm{MV}_{2}(\mathrm{M}=\mathrm{Zr}$, Hf, or Ta)}

The total energy vs. volume curves for the $\mathrm{C} 15$ Laves compounds, $\mathrm{MV}_{2}(\mathrm{M}=\mathrm{Zr}$, Hf, or $\mathrm{Ta}$ ) were obtained. Based on these curves, the calculated lattice parameter (a) and bulk modulus (B) corresponding to the energy minimum obtained from these curves are tabulated in Table 1. It can be seen from Table 1 that the theoretical lattice parameter (a) is just 1-2\% off of the experimental value $\left(a_{0}\right)$, which shows good agreement between the theoretical and the experimental results.

TABLE 1 The lattice parameters and bulk moduli of $\mathrm{C} 15 \mathrm{MV}_{2}(\mathrm{M}=\mathrm{Zr}$, Hf, or Ta).

\begin{tabular}{cccc}
\hline C15 Phases & $\mathrm{a}_{0}(\AA)$ & $\mathrm{a}(\AA)$ & $\mathrm{B}(\mathrm{GPa})$ \\
\hline \hline $\mathrm{ZrV}_{2}$ & 7.4440 & 7.5467 & 162 \\
$\mathrm{HfV}_{2}$ & 7.4239 & 7.4740 & 172 \\
$\mathrm{TaV}_{2}$ & 7.1570 & 7.2977 & 218 \\
\hline
\end{tabular}


The energy-band structures of the $\mathrm{C} 15 \mathrm{MV}_{2}(\mathrm{M}=\mathrm{Zr}$, Hf, or Ta) along the highsymmetry directions in the Brillouin zone were obtained at the equilibrium configurations [23]. There is a large number of flat energy bands at or below the Fermi level, $\mathrm{E}_{\mathrm{F}}$, as is reflected also in the density of states. Figure 4 shows the detailed features of this band structure near the Fermi level and the X-point of the IBZ. There is, indeed, a double degeneracy of electron energy levels with a linear dispersion relationship in the vicinity of the X-point, which is related to the special symmetry Fd3m of the C15 structure [19]. However, we can see from Fig. 4 that the Fermi surface does not pass through the X-point of the IBZ exactly, but passes near the Xpoint so that there is an energy gap, $\Delta \varepsilon$, between the double degeneracy point and the Fermi level. This is not consistent with the assumption by Izyumov et al. [19]. The $\Delta \varepsilon$ values for C15 $\mathrm{MV}_{2}(\mathrm{M}=\mathrm{Zr}, \mathrm{Hf}$, or Ta) are listed in Table 2

TABLE 2 The energy gap, $\Delta \varepsilon$, of $\mathrm{C} 15 \mathrm{MV}_{2}(\mathrm{M}=\mathrm{Zr}$, Hf, or Ta).

\begin{tabular}{ccc}
\hline C15 Phases & $\Delta \varepsilon($ mRyd $)$ & $\Delta \varepsilon(\mathrm{K})$ \\
\hline \hline $\mathrm{ZrV}_{2}$ & 5.0 & 781 \\
$\mathrm{HfV}_{2}$ & 4.8 & 750 \\
$\mathrm{TaV}_{2}$ & 6.0 & 1000 \\
\hline
\end{tabular}

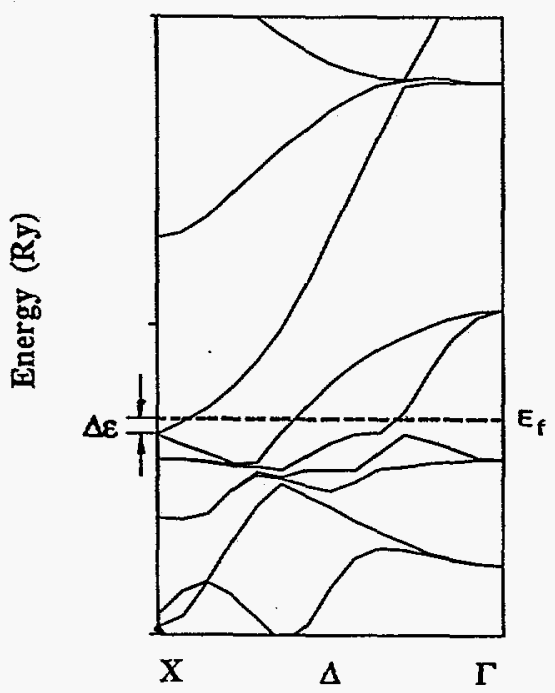

Figure 4. Detailed features of the band structure near the Fermi level for $\mathrm{C} 15 \mathrm{MV}_{2},(\mathrm{M}=\mathrm{Zr}, \mathrm{Hf}$, or Ta), showing the double degeneration with a linear dispersion relation and energy gap $(\Delta \varepsilon)$.

In this case, it can be shown that the electronic contribution to the shear modulus, $c_{44}^{e}$, follows the equation [23]:

$$
c_{44}^{(e)} \propto-\int_{0}^{\infty} \frac{d \varepsilon}{\varepsilon} e^{\frac{\Delta \varepsilon}{T}}\left(\frac{e^{\frac{\varepsilon}{2 T}}}{e^{-\frac{\varepsilon}{2 T}}+e^{\frac{\Delta \varepsilon}{T}} e^{\frac{\varepsilon}{2 T}}}-\frac{e^{-\frac{\varepsilon}{2 T}}}{e^{\frac{\varepsilon}{2 T}}+e^{\frac{\Delta \varepsilon}{T}} e^{-\frac{\varepsilon}{2 T}}}\right)
$$

Based on this formula, if the Fermi surface passes exactly through the double degenerate point $(\Delta \varepsilon=0), c_{44}^{e}$ obeys the following equation:

$$
c_{44}^{(e)} \propto-\int_{0}^{\omega} \frac{d \varepsilon}{\varepsilon} \tanh \frac{\varepsilon}{2 T}
$$


The integral on the right hand side of the above equation gives the following temperature dependence of $c_{44}^{e}$ :

$$
c_{44}^{(e)} \propto-\sum_{n=1}^{\infty} B_{n} \frac{(-1)^{n-1} 2^{2 n}\left(2^{2 n}-1\right)}{(2 n-1)(2 n) !}\left(\frac{\omega}{2 T}\right)^{2 n-1}
$$

$c_{44}^{e}$ is negative and anomalous in the entire temperature region. However, if the Fermi surface passes near the double degenerate point with an energy gap $(\Delta \varepsilon)$, as $\mathrm{C} 15 \mathrm{MV}_{2}(\mathrm{M}=\mathrm{Zr}$, Hf, or Ta) has, $c_{44}^{e}$ is negative but anomalous only at high temperatures, depending on the value of $\Delta \varepsilon$, as shown in Fig. 5.

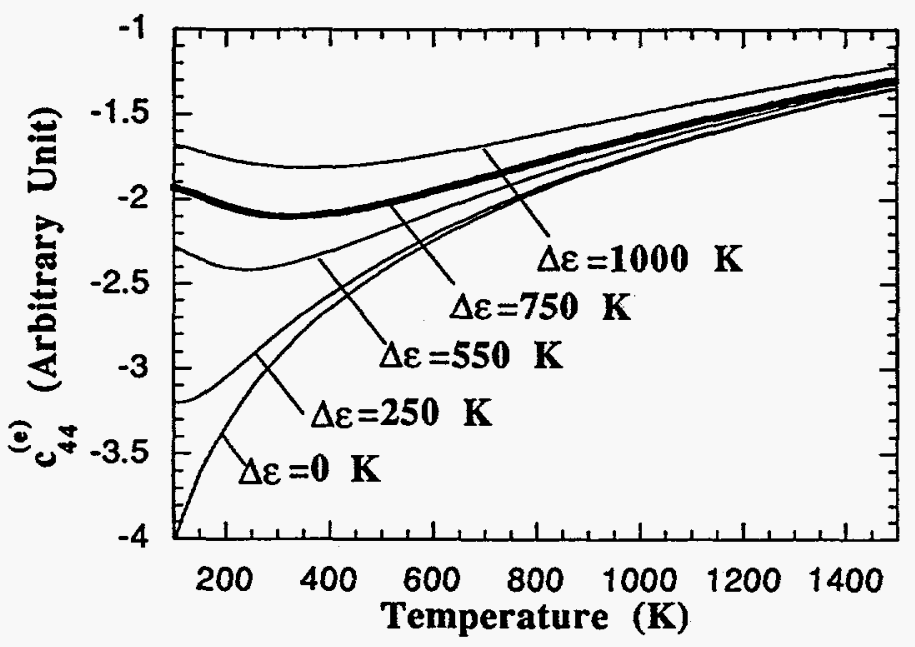

Figure 5. Temperature dependence of $c_{44}^{e}$ for different $\Delta \varepsilon$. The thick line corresponds to the case of $\mathrm{C} 15 \mathrm{HfV}_{2}$.

These results suggest that for $\mathrm{C} 15 \mathrm{HfV}_{2}$ the electronic contribution to the shear modulus, $c_{44}^{e}$, is negative and anomalous at high temperatures $(\mathrm{T}>300 \mathrm{~K})$, i.e. $c_{44}^{e}$ increases with increasing temperature at high temperatures. This can explain the experimentally observed anomalous behavior of shear modulus for $\mathrm{C} 15 \mathrm{HfV}_{2}$ at high temperatures [17]. It is also suggested based on these arguments that the V-shaped low temperature behavior of $\mathrm{c}_{44}$ for C15 $\mathrm{HfV}_{2}$ is not due to the electronic effect because $c_{44}^{e}$ shows no stiffening in the low temperature region and, therefore, the low temperature phase transformation is not caused by the cancellation of the negative electronic contribution to the shear modulus with the corresponding positive modulus due to ion-ion interaction, $c_{44}^{i}$, as suggested by Izyumov et al. [18].

A further detailed analysis for the density of states and the Fermi surface of $\mathrm{C} 15 \mathrm{MV}_{2}(\mathrm{M}=\mathrm{Zr}$, Hf, or Ta) suggests a new mechanism for the phase instability of $\mathrm{C} 15$ $\mathrm{HfV}_{2}$.

The density of states (DOS) of C15 $\mathrm{MV}_{2}(\mathrm{M}=\mathrm{Zr}, \mathrm{Hf}$, or $\mathrm{Ta})$ is shown in Fig. 6 . It can be seen that for these systems, the density of states at the Fermi level, $N\left(E_{F}\right)$, is very large, as listed in Table 3 . This results in a very strong electron-phonon coupling due to the contribution of a large number of electrons at the Fermi level.

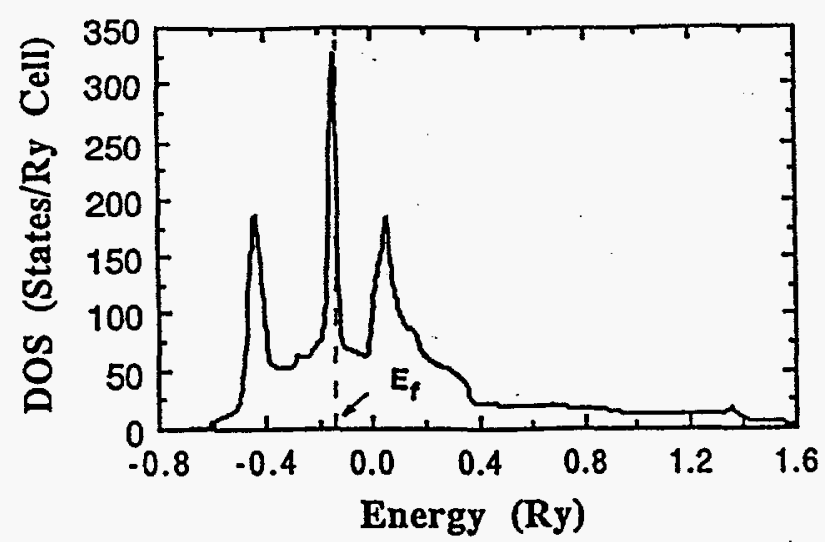

Figure 6. DOS of C15 $\mathrm{MV}_{2}(\mathrm{M}=\mathrm{Zr}$, Hf, or Ta). 
TABLE $3 \mathrm{~N}\left(\mathrm{E}_{\mathrm{F}}\right)$ of C15 $\mathrm{MV}_{2}(\mathrm{M}=\mathrm{Zr}$, Hf, or Ta).

\begin{tabular}{cc}
\hline C15 Phases & $N\left(E_{F}\right)$ (States/ Rdy Cell) \\
\hline \hline $\mathrm{ZrV}_{2}$ & 184.96 \\
$\mathrm{HfV}_{2}$ & 196.84 \\
$\mathrm{TaV}_{2}$ & 176.21 \\
\hline
\end{tabular}

The Fermi surfaces of $\mathrm{C} 15 \mathrm{MV}_{2}(\mathrm{M}=\mathrm{Zr}$, Hf, or Ta) are shown in Fig. 8. It can be seen from Fig. 8 that $\mathrm{C} 15 \mathrm{HfV}_{2}$ and $\mathrm{ZrV}_{2}$ have Fermi surface nesting with a $\mathbf{q} / /<100>$ while $\mathrm{C} 15 \mathrm{TaV}_{2}$ does not. The Fermi surface nesting results in a very large electronic susceptibility, which induces an electronic charge density wave (along $<100\rangle$ with a wavelength $\lambda \sim 1 / \mathrm{q}$ ). This electronic charge density wave may induce a structural modulation through the strong electronphonon interaction due to the large $N\left(E_{F}\right)$ for $C 15 \mathrm{HfV}_{2}$ and $\mathrm{ZrV}_{2}$ at low temperatures. We suggest that this could be the mechanism for the structural transformation in $\mathrm{C} 15 \mathrm{HfV}_{2}$, as described above, and in $\mathrm{C} 15 \mathrm{ZrV}_{2}$ [15-16]. In fact, both the susceptibility increase and the partial phonon softening have been experimentally observed around the phase transformation temperature for $\mathrm{HfV}_{2}$ [17]. We believe that the low temperature structural instability for C15 transition metal Laves phases is driven by phonon softening $(\omega(\mathbf{q}), \mathbf{q} / /<001>)$, resulting from the strong electron-phonon coupling due to the large $N\left(E_{F}\right)$ and Fermi surface nesting.



(a)

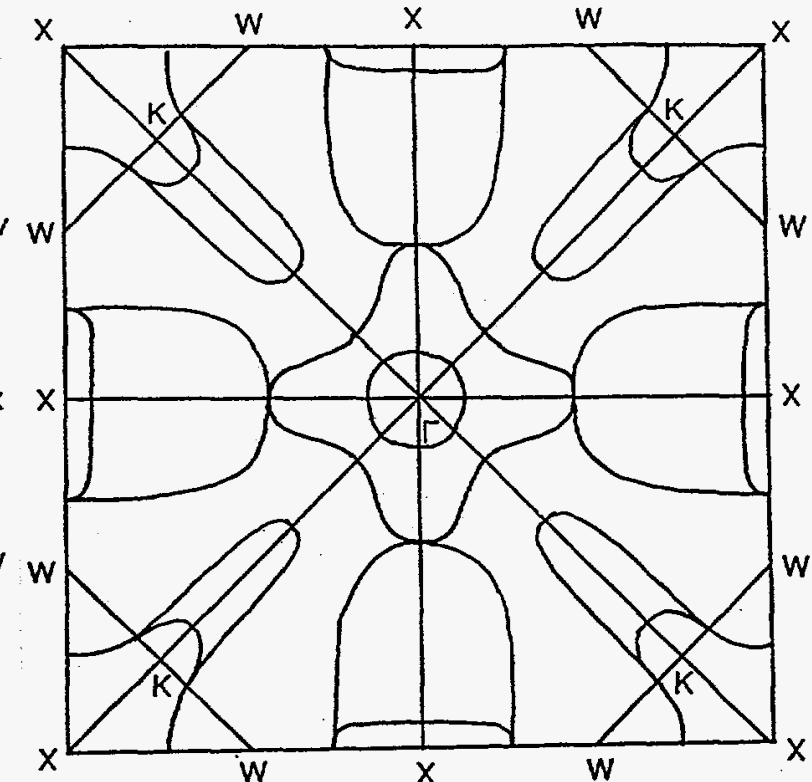

(b)

Figure 7. The Fermi surfaces of (a) $\mathrm{C} 15 \mathrm{HfV}_{2}$ and $\mathrm{ZrV}_{2}$, showing the Fermis surface nesting with $q / /<100>$, and (b) $\mathrm{C} 15 \mathrm{TaV}_{2}$, showing the disappearance of the Fermi surface nesting.

On the other hand, for $\mathrm{C} 15 \mathrm{TaV}_{2}$, because Ta provide one more valence electron than $\mathrm{Hf}$ does, the Fermi level increases correspondingly. Based on the rigid-band assumption, this results in two effects: (a) the $\mathrm{N}\left(\mathrm{E}_{\mathrm{F}}\right)$ for $\mathrm{C} 15 \mathrm{TaV}_{2}$ is decreased, as shown in Table 3, and (b) the shape of the Fermi surface is dramatically changed, resulting in the disappearance of the Fermi surface nesting, due to the flattened bands near the Fermi level, as mentioned above. Due to the relatively weak electron-phonon coupling and the lack of Fermi surface nesting, the low temperature phase transformation could not occur in $C 15 \mathrm{TaV}_{2}$. In fact, this has been experimentally proved, as shown in Fig. 8. Based on this argument, the stabilization of the $\mathrm{C} 15$ structure in $\mathrm{HfV}_{2}+\mathrm{Nb}$ by $\mathrm{Nb}$ alloying can be understood in the same way: $\mathrm{Nb}$ provides one more electrons than $\mathrm{Hf}$ does and the substitution of $\mathrm{Hf}$ site by $\mathrm{Nb}$ increases the Fermi level, resulting in a smaller $\mathrm{N}\left(\mathrm{E}_{\mathrm{F}}\right)$ the disappearance of Fermi surface nesting. Therefore, there is no 
such a transformation in $\mathrm{C} 15 \mathrm{HfV}_{2}+\mathrm{Nb}$ induced by phonon softening $(\omega(\mathbf{q}), \mathbf{q} / /<001>)$, resulting from the strong electron-phonon coupling due to the large $N\left(E_{F}\right)$ and Fermi surface nesting.

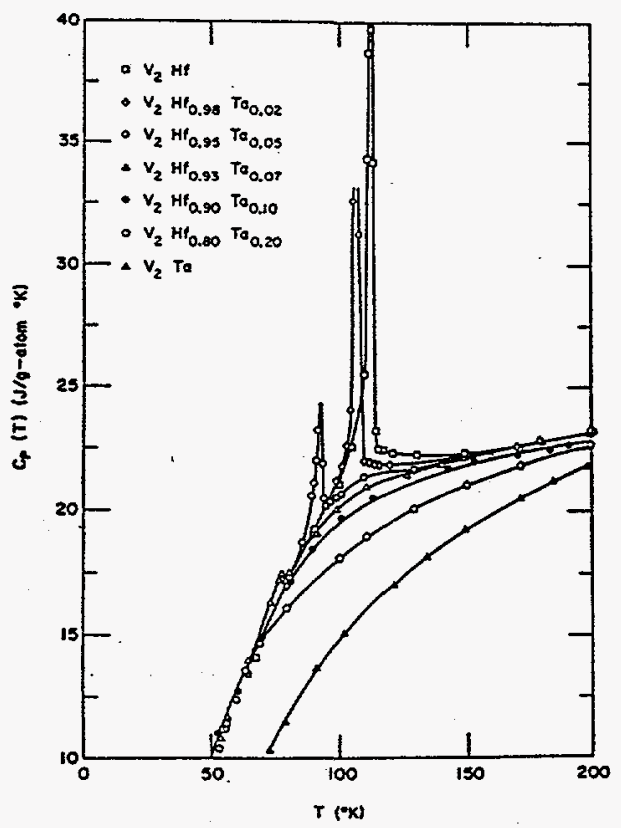

Figure 8 . Heat capacity vs. temperature in the vicinity of the lattice transformation temperature for $\mathrm{C} 15\left(\mathrm{Hf}_{1-\mathrm{x}} \mathrm{Ta}_{\mathrm{x}}\right) \mathrm{V}_{2}$ compounds (from Ref. [16]), showing that there is no low temperature phase transformation in $\mathrm{C} 15 \mathrm{TaV}_{2}$.

It is necessary to point out that the recently-finished more precise total energy and electronic structure calculations, full-potential LMTO calculations, yield the similar results for $\mathrm{C} 15 \mathrm{HfV}_{2}$ as mentioned above [24]. Therefore, the atomic sphere approximation in the present calculations may not bring ambiguity in the above arguments for the analysis of the phases stability and elasticity of C15 transition metal Laves phases.

\section{Conclusions}

Based on the combined experimental and theoretical study for the phase stability and elasticity of $\mathrm{C} 15 \mathrm{HfV}_{2}+\mathrm{Nb}$, we conclude:

1. The phase stability of the $\mathrm{C} 15$ Laves compounds $\mathrm{HfV}_{2}+\mathrm{Nb}$ has been investigated. The binary $\mathrm{C} 15$ Laves phase $\mathrm{HfV}_{2}$ is unstable at low temperatures. A structural phase transformation occurs at about $115 \mathrm{~K}$. Nb ternary alloying can reduce the transition temperature and heat absorption in the structural transformation of the $\mathrm{C} 15$ Laves phase $\mathrm{HfV}_{2}+\mathrm{Nb}$. A small amount of $\mathrm{Nb}$ doping ( $\mathrm{Nb}$ at.\%>2-3) can eliminate this phase transformation. This means that the role played by the $\mathrm{Nb}$ ternary alloying in $\mathrm{HfV}_{2}$ is to stabilize the structure of $\mathrm{C} 15$. We suggest that low temperature structural instability of $\mathrm{C} 15 \mathrm{HfV}_{2}$ and $\mathrm{ZrV}_{2}$ is induced by phonon softening, resulting from a large $\mathrm{N}\left(\mathrm{E}_{\mathrm{F}}\right)$ and Fermi surface nesting. The lack of structural instability of $\mathrm{C} 15 \mathrm{TaV}_{2}$ and $\mathrm{C} 15 \mathrm{HfV}_{2}+\mathrm{Nb}$ is due to the disappearance of Fermi surface nesting and a smaller $\mathrm{N}\left(\mathrm{E}_{\mathrm{F}}\right)$, because of the increase in valence electrons.

2. Anomalous elastic properties exist in both binary and ternary C15 Laves compounds $\mathrm{HfV}_{2}+\mathrm{Nb}$. For ternary $\mathrm{C} 15$ compounds, the shear and Young's moduli increase with increasing temperature, the bulk modulus is virtually constant, and the Poisson's ratio is very high and decreases with increasing temperature. The special electronic structure at the X-point of IBZ near the Fermi level may be one of the reasons for the C15 anomalous temperature dependence of the shear modulus at high temperatures.

3. The low temperature structural instability of $\mathrm{C} 15 \mathrm{HfV}_{2}$ and $\mathrm{ZrV}_{2}$ may not be directly related to the anomalous shear modulus $\left(c_{44}\right)$ induced by the electronic effect. 
F. Chu is grateful to Prof. Vitek for useful discussions and comments. F. Chu is supported by a Director-Funded Post-Doctoral Fellowship at Los Alamos National Laboratory. This research has been supported by the National Aeronautics and Space Administration and the Office of Naval Research (Grant No. N00014-91-J-1165P02), and the DOE-OBES at Los Alamos National Laboratory.

\section{$\underline{\text { References }}$}

1. D. P. Pope and C. T. Liu, "Strength and Ductility of Intermetallic Compounds", in Superalloys, Supercomposites and Superceramics, ed. by J. K. Tien, and T. Caulfield, (Academic Press, New York) (1989), 583-624.

2. D. P. Pope and F. Chu, "Deformation of a C15 Laves Phase: Twinning and Synchroshear", in Structural Intermetallics, ed. by R. Darolia, J. J. Lewandowski, C. T. Liu, P. L. Martin, D. B. Miracle, and M. V. Nathal, (TMS Publication, Warrendale, PA), (1993), 637-646.

3. T. E. Mitchell, R. G. Castro, J. J. Petrovic, S. A. Maloy, O. Unal, and M. M. Chadwick, "Dislocations, twins, grain boundaries and precipitates in MoSi2", Mater. Sci. \& Eng., A155, (1992), 241-250.

4. J. D. Livingston and E. L. Hall, "Room temperature deformation in a Laves phase", J. mater. Res. 5(1), (1990), 5-8.

5. F. Chu and D. P. Pope, "Deformation twinning in intermetallic compounds-the dilemma of shear vs. shuffles", Mater. Sci. \& Eng., A170, (1993), 39-47.

6. M. Takeyama, and C. T. Liu, "Microstructures and michanical properties of Laves phases alloy based on $\mathrm{Cr}_{2} \mathrm{Nb}$ ", Mater. Sci. \& Eng., A132 (1991), 61-66.

7. D. J. Thoma and J. H. Perepezko, "An experimental evaluation of the phase relationships and solubilities in the Nb-Cr system", Mater. Sci. \& Eng., A156, (1992), 97 108.

8. K. S. Kumar and D. B. Miracle, "Microstructural evolution and mechanical properties of a Cr-Cr2Hf alloy", Intermetallics 2, (1994), 257-274.

9. K. S. Kumar and P. M. Hazzledine, "The $\mathrm{C} 14$ to $\mathrm{C} 15$ transformation in $\mathrm{Cr}_{2} \mathrm{Hf}$ ", in High Temperature Ordered Intermetallic Alloys-VI, ed. by J. Horton, I. Baker, S. Hanada and R. D. Noebe, Mat. Res. Soc. Symp. Proc., Vol. 364, (1995).

10 Y. Liu, S. M. Allen and J. D. Livingston, "Deformation Mechanisms in a Laves Phase", in High Temperature Intermetallic Alloys-V, ed. I. Baker, R. Darolia, J. D. Whittenberger and M. H. Yoo, Mat. Res. Soc. Symp. Proc., Vol. 364, (1993), 203-208.

11. K. C. Chen, S. M. Allen and J. D. Livingston, "Morphology, Deformation, and Defect Structure of $\mathrm{TiCr}_{2}$ in Ti-Cr Alloy", ed. I. Baker, R. Darolia, J. D. Whittenberger and M. H. Yoo, Mat. Res. Soc. Symp. Proc., Vol. 364, ( 1993), 373-378.

12. D. G. Pettifor, "Electron theory of metals", in Physical Metallurgy, ed. by R. W. Cahn and P. Haasen, North-Holland Physics Publishing, (Amsterdam, 1983), 73-152.

13. Y. Ohta and D. G. Pettifor, "Size versus electronic factors in transition metal Laves phase stability", J. Phys. Condens. Matter 2, (1990), 8189-8194.

14. M. Sluiter and P. E. A. Turchi, "Phase stability in Ti-V and Ti-Cr alloys: a theoretical investigation", Phys. Rev. B 43, (1991), 12251-12266.

15. A. C. Lawson and W. H. Zachariasen, "Low temperature lattice transformation of HfV 2 ", Phys. Lett., 38A(1), (1965), 1-1.

16. T. R. Finlayson, E. J. Lanston, M. A. Simpson, E. E. Gibbs, and T. F. Smith, "Elastic properties of (Hf,Zr)V 2 superconducting compounds", J. Phys. F., 8, (1978), 2269-2278.

17. J. W. Hafstrom, G. S. Knapp, and A. T. Aldred, "Electronic and lattice properties of $\mathrm{V}_{2} \mathrm{Hf}_{1-\mathrm{x}} \mathrm{Ta}_{\mathrm{x}} \mathrm{C}-15$ superconductors", Phys. Rev. B 17, (1978), 2892-2900.

18. A. S. Balankin and D. M. Skorov, "Anomalies of the elastic moduli of the Laves phase $\mathrm{ZrV}_{2}$ and $\mathrm{HfV}_{2}$ at high temperatures", Sov. Phys. Solid State, 24, (1982), 681-682.

19. Y.A. Izyumov, V. Y. Naysh, and V. N. Syromyatnikov, "Concerning lattice anomalies in high temperature superconductive compounds of the A15 and C15 type", Fiz. Metal. Metalloved., 39, (1975), 455-460. 
20. F. Chu and D. P. Pope, "The Laves phase field in the Hf-V-Nb system", Scripta Met. et - Mater., 26, (1992), 399-404.

21. A. Migliori, J. L. Sarrao, W. M. Visscher, T. M. Bell, Ming Lei, Z. Fisk and R. G. Leisure, "Resonant ultrasound spectroscopic techniques for measurements of the elastic moduli of solids", Physica B, 183, (1993), 1-24.

22. F. Chu, Ming Lei, A. Migliori, S. P. Chen and T. E. Mitchell, Anomalous elastic properties in a C15 Laves-pase compound", Phil. Mag. B 70, (1994), 867-880.

23. F. Chu, M. Sob, R. Siegl, T. E. Mitchell and S. P. Chen, Total energy and electronic structure calculations of $\mathrm{C} 15$ Laves-phase compounds $\mathrm{MV}_{2}(\mathrm{M}=\mathrm{Zr}$, Hf or Ta): elastic properties", Phil. Mag. B 70, (1994), 881-892.

24. A. H. Omenci, F. Chu, T. E. Mitchell, S. P. Chen, D. J. Thoma, J. M. Wills and R. C. Albers, to be published. 